\title{
Manifolds admitting both strongly irreducible and weakly reducible minimal genus Heegaard splittings
}

\author{
Tsuyoshi Kobayashi and Yo'AV RIECK
}

\begin{abstract}
We construct infinitely many manifolds admitting both strongly irreducible and weakly reducible minimal genus Heegaard splittings. Both closed manifolds and manifolds with boundary tori are constructed.
\end{abstract}

\section{Introduction}

The pioneering work of Casson and Gordon [1] shows that a minimal genus Heegaard splitting of an irreducible, non-Haken three-manifold is necessarily strongly irreducible; by contrast, Haken [2] showed that a minimal genus (indeed, any) Heegaard splitting of a composite three-manifold is necessarily reducible, and hence weakly reducible. The following question of Moriah [9] is therefore quite natural:

Question 1.1 ([9, Question 1.2]). Can a three-manifold $M$ have both weakly reducible and strongly irreducible minimal genus Heegaard splittings?

We answer this question affirmatively:

Theorem 1.1. There exist infinitely many closed, orientable threemanifolds of Heegaard genus 3, each admitting both strongly irreducible and weakly reducible minimal genus Heegaard splittings.

Theorem 1.1 is proved in Section 3. In Remark 3.2 we offer a strategy to generalize Theorem 1.1 to construct examples of genus $g$, for each $g \geq$ 3 ; it is easy to see that no such examples can exist if $g<3$. In Section 4 , we give examples of manifolds with one, two or three torus boundary components, each admitting both strongly irreducible and weakly reducible minimal genus Heegaard splittings. Moreover, for each manifold with two boundary components, we construct four minimal genus Heegaard surfaces, two weakly reducible, one separating the boundary components and one that 
does not, and similarly two strongly irreducible minimal genus Heegaard surfaces. For a precise statement, see Theorem 4.1.

In an effort to keep this article short, we refer the readers to Section 3 of [7] for definitions and background material. Unless otherwise stated, we follow the notations of that paper.

\section{Preliminaries}

\subsection{Constructing strongly irreducible Heegaard splittings}

In this section, we introduce a method for constructing strongly irreducible Heegaard splittings using two-bridge link exteriors; this is taken out of [6].

\section{Definition 2.1.}

(1) A two-string tangle $\left(B^{3} ; t_{1}, t_{2}\right)$ is a pair of three-ball $B^{3}$ and two disjoint arcs $t_{1}$ and $t_{2}$ properly embedded in $B^{3}$.

(2) A tangle is called two-string trivial tangle if it is homeomorphic (as a triple) to $\left(D^{2} \times[0,1] ;\{p\} \times[0,1]:\{q\} \times[0,1]\right)$, where $D^{2}$ is a two-disk and $p$ and $q$ are two distinct points in $\operatorname{int}\left(D^{2}\right)$.

For $Y \subset X$ with $\operatorname{dim} X=\operatorname{dim} Y$, we denote the frontier of $Y$ in $X$ by $\operatorname{Fr}_{X}(Y)$. Let $\left(B^{3} ; t_{1}, t_{2}\right)$ be a two-string trivial tangle. Let $H=\operatorname{cl}\left(B^{3} \backslash\right.$ $\left.\left(N\left(t_{1}\right) \cup N\left(t_{2}\right)\right)\right)$ and $A_{i}=\operatorname{Fr}_{B^{3}}\left(N\left(t_{i}\right)\right), i=1,2$. Note that $H$ is a genus two handlebody, $A_{1}$ and $A_{2}$ are annuli in $\partial H$ and the pair $\left\{A_{1}, A_{2}\right\}$ is primitive in $H$ (see Figure 1), i.e., there exist pairwise disjoint meridian disks $\Delta_{1}$, $\Delta_{2} \subset H$ so that

(1) $\Delta_{i} \cap A_{i}$ is an essential arc in $A_{i}(i=1,2)$ and

(2) $\Delta_{1} \cap A_{2}, \Delta_{2} \cap A_{1}=\emptyset$.
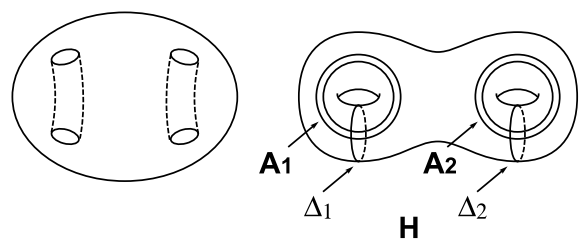

Figure 1: Exterior of a trivial tangle. 
A link $L \subset S^{3}$ is called a two-bridge link, if it can be expressed as the union of two two-string trivial tangles; more precisely, if $\left(S^{3} ; L\right)=\left(B ; t_{1}, t_{2}\right) \cup$ $\left(B^{\prime} ; t_{1}^{\prime}, t_{2}^{\prime}\right)$, where $\left(B ; t_{1}, t_{2}\right)$ and $\left(B^{\prime} ; t_{1}^{\prime}, t_{2}^{\prime}\right)$ are two-string trivial tangles, $B \cap B^{\prime}=\partial B=\partial B^{\prime}$ and $L=\left(t_{1} \cup t_{1}^{\prime}\right) \cup\left(t_{2} \cup t_{2}^{\prime}\right)$. Note that in this paper by a two-bridge link we always mean a two-component link, and not a twobridge knot.

Let $\left(H, A_{1} \cup A_{2}\right)$ be as above and $\left(H^{\prime}, A_{1}^{\prime} \cup A_{2}^{\prime}\right)$ be a copy of $\left(H, A_{1} \cup\right.$ $\left.A_{2}\right), P=\operatorname{cl}\left(\partial H \backslash\left(A_{1} \cup A_{2}\right)\right)$, and similarly, $P^{\prime}=\operatorname{cl}\left(\partial H^{\prime} \backslash\left(A_{1}^{\prime} \cup A_{2}^{\prime}\right)\right)$. Let $L$ be a two-bridge link. Then we see from the above that there exists a homeomorphism $h: P \rightarrow P^{\prime}$ such that $E(L)$, the exterior of $L$, is homeomorphic to $H \cup_{h} H^{\prime}$ and $\partial E(L)=\left(A_{1} \cup A_{1}^{\prime}\right) \cup\left(A_{2} \cup A_{2}^{\prime}\right)$, so that $\partial A_{i}$ and $\partial A_{i}^{\prime}$ are meridian curves $(i=1,2)$. The image of $P=P^{\prime}$ in $E(L)$ is called a bridge sphere.

Let $N$ be a (possibly disconnected) orientable, irreducible, $\partial$-irreducible three-manifold such that $\partial N$ consists of two tori $T_{1}$ and $T_{2}$ and each component of $N$ has non-empty boundary (hence, $N$ consists of at most two components). Suppose that there exists a three-dimensional sub-manifold $R \subset N$ such that

(1) each component of $R$ is a handlebody and $\operatorname{Fr}_{N}(R)$ is incompressible in $N$;

(2) $T_{i} \cap R(i=1,2)$ consists of an annulus, say $\mathcal{A}_{i}$, such that

(a) $\mathcal{A}_{i}$ is incompressible in $N$ and

(b) $\mathcal{A}_{i}$ is $\partial$-incompressible in $R$ (i.e., there does not exist a disk properly embedded in $R$ that intersects $\mathcal{A}_{i}$ in an essential arc);

(3) each component of $\operatorname{cl}(N \backslash R)=R^{\prime}$ is a handlebody such that $T_{i} \cap R^{\prime}$ $(i=1,2)$ consists of an annulus, say $\mathcal{A}_{i}^{\prime}$ satisfying

(a) $\mathcal{A}_{i}^{\prime}$ is incompressible in $N$ and

(b) $\mathcal{A}_{i}^{\prime}$ is $\partial$-incompressible in $R^{\prime}$.

With notations as above, let $M$ be the three-manifold obtained from $E(L)$ and $N$ by identifying their boundary by an orientation reversing homeomorphism $\partial N \rightarrow \partial(E(L))$ such that $\mathcal{A}_{i}\left(\mathcal{A}_{i}^{\prime}\right.$ resp. $)$ is mapped to $A_{i}\left(A_{i}^{\prime}\right.$ resp.). Let $V=H \cup R \subset M$ and similarly $V^{\prime}=H^{\prime} \cup R^{\prime} \subset M$. Since $A_{1} \cup A_{2}$ is primitive in $H, H$ is obtained by attaching a single one-handle to two solid tori, with $A_{1}$ a longitudinal annulus on one solid torus and $A_{2}$ a longitudinal annulus on the other. Hence, gluing $H$ to $R$ along $A_{1} \cup A_{2}$ is equivalent to attaching a single one-handle to $R$, and similarly for $H^{\prime}$ and $R^{\prime}$. We see that $V$ ( $V^{\prime}$ resp.) is a handlebody obtained from $R$ ( $R^{\prime}$ resp.) by attaching 


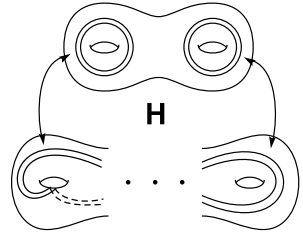

$\mathbf{R}$

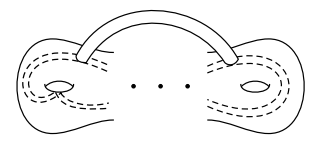

V

Figure 2: $V$ is obtained from $R$ by attaching a 1-handle.

a one-handle (see Figure 2), and therefore $V \cup V^{\prime}$ is a Heegaard splitting of $M$. For this Heegaard splitting the following holds:

Proposition 2.1. With notations as above, if $L$ is not the trivial link or the Hopf link, then the Heegaard splitting $V \cup V^{\prime}$ is strongly irreducible.

Sketch of proof. Proposition 2.1 is identical to Proposition 3.1 of [6] and the proof can be found there. For the convenience of the readers, we sketch it here. Let $D \subset V$ and $D^{\prime} \subset V^{\prime}$ be a pair of meridian disks. Minimize the intersection of $D$ with $A_{1} \cup A_{2}$ and the intersection of $D^{\prime}$ with $A_{1}^{\prime} \cup A_{2}^{\prime}$. By symmetry, we have the following three cases:

(1) $D \cap\left(A_{1} \cup A_{2}\right)=\emptyset$ and $D^{\prime} \cap\left(A_{1}^{\prime} \cup A_{2}^{\prime}\right)=\emptyset$,

(2) $D \cap\left(A_{1} \cup A_{2}\right)=\emptyset$ and $D^{\prime} \cap\left(A_{1}^{\prime} \cup A_{2}^{\prime}\right) \neq \emptyset$,

(3) $D \cap\left(A_{1} \cup A_{2}\right) \neq \emptyset$ and $D^{\prime} \cap\left(A_{1}^{\prime} \cup A_{2}^{\prime}\right) \neq \emptyset$.

In the first case, $D$ (resp. $\left.D^{\prime}\right)$ is the meridian disk of the tangle $\left(B ; t_{1}, t_{2}\right)$ (resp. $\left.\left(B^{\prime} ; t_{1}^{\prime}, t_{2}^{\prime}\right)\right)$; since $L$ is not the trivial link, $D$ intersects $D^{\prime}$ more than twice. In the second case, $D$ is the meridian disk of the tangles $\left(B ; t_{1}, t_{2}\right)$. Consider an outermost disk on $D^{\prime}$, say $\delta^{\prime}$. Note that $\delta^{\prime} \subset H^{\prime}$. If the arc of $\delta^{\prime}$ on $A_{1}^{\prime}$ or $A_{2}^{\prime}$ (say the latter) is inessential, we can surger $D^{\prime}$ along the disk component of $A_{2}^{\prime} \backslash \delta^{\prime}$ to obtain a meridian disk of the tangle $\left(B^{\prime} ; t_{1}^{\prime}, t_{2}^{\prime}\right)$; the proof now is the same as the first case. Else, $\delta^{\prime}$ gives a boundary compression for $A_{1}^{\prime}$ or $A_{2}^{\prime}$. Again, since $L$ is not the trivial link, we see that $\left|D^{\prime} \cap D\right| \geq$ $\left|\delta^{\prime} \cap D\right|>1$.

In the third case, we consider outermost disks, $\delta$ on $D$, and $\delta^{\prime}$ on $D^{\prime}$. If the arc of $\delta$ on $A_{1}$ or $A_{2}$ is inessential, or the arc of $\delta^{\prime}$ on $A_{1}^{\prime}$ or $A_{2}^{\prime}$ is inessential, then arguments similar to the above work. Suppose $\delta$ on $A_{1}$ or $A_{2}$ and $\delta^{\prime}$ on $A_{1}^{\prime}$ or $A_{2}^{\prime}$ are essential. Since $L$ is not the trivial link or the Hopf link, we see that $\left|D^{\prime} \cap D\right| \geq\left|\delta^{\prime} \cap \delta\right| \geq 1$. 


\subsection{Spines of amalgamated Heegaard splittings.}

A spine of a compression body $C$ is a graph $\lambda$ embedded in $C$ so that $C \backslash$ $\left(\lambda \cup \partial_{-} C\right)$ is homeomorphic to $\partial_{+} C \times(-\infty, 0]$. Let $C \cup C^{\prime}$ be a Heegaard splitting of a manifold $M$; a graph $\Gamma \subset M$ is a spine for $C$ if there exists an ambient isotopy of $M$, so that the image of $\Gamma$ after this isotopy is contained in $C$ as a spine. Simultaneous spines of $C \cup C^{\prime}$ are two disjointly embedded graphs $\Gamma, \Gamma^{\prime} \subset M$, so that after an ambient isotopy of $M$, the image of $\Gamma$ ( $\Gamma^{\prime}$ resp.) is contained in $C\left(C^{\prime}\right.$ resp.) as a spine.

For the definition of amalgamation of Heegaard splittings, see [10].

Proposition 2.2. Let $M_{1}$ and $M_{2}$ be manifolds so that $\partial M_{1}$ and $\partial M_{2}$ are connected and homeomorphic. For $i=1,2$, let $H_{i} \cup C_{i}$ be Heegaard splittings of $M_{i}$, where $H_{i}$ is a handlebody and $C_{i}$ a compression body. Let $\mu_{i}$ (resp. $\lambda_{i}$ ) be a spine of $H_{i}$ (resp. $\left.C_{i}\right)$. Let $M$ be a manifold obtained by gluing $M_{1}$ and $M_{2}$ along their boundaries. Let $H \cup H^{\prime}$ be the amalgamation of $H_{1} \cup C_{1}$ and $\mathrm{H}_{2} \cup \mathrm{C}_{2}$.

Then there exist simultaneous spines of $H \cup H^{\prime}$ so that $\mu_{1} \cup \lambda_{2}$ is contained in a spine of $H$ or $H^{\prime}$, and $\mu_{2} \cup \lambda_{1}$ is contained in a spine of the other.

Proof. We denote the image of $\partial M_{i}$ in $M$ by $F$, the image of $\mu_{i}$ in $M$ by $\mu_{i}$ and the image of $\lambda_{i}$ in $M$ by $\lambda_{i}$. By transversality, we assume as we may that $\lambda_{1} \cap \lambda_{2}=\emptyset$. The Heegaard surface that gives amalgamation of $H_{1} \cup C_{1}$ and $H_{2} \cup C_{2}$ is given by tubing $F$ along $\lambda_{1}$ into $M_{1}$ and along $\lambda_{2}$ into $M_{2}$, see Figure 3 (this figure is based on Schultens' [10, Figure 3]). Note that the intersection of $F$ and the amalgamated Heegaard surface is not transverse.

We may suppose that $\mu_{1} \cup \lambda_{2}$ is contained in $H$ and $\mu_{2} \cup \lambda_{1}$ is contained in $H^{\prime}$. By compressing $H$ along the disks $\operatorname{cl}(\operatorname{int}(H) \cap F)$, we obtain two handlebodies. One handlebody is isotopic to $H_{1}$ and so we may take $\mu_{1}$ as its spine. The other handlebody contains $\lambda_{2}$ and admits a deformation retract onto it; moreover, $\lambda_{2}$ intersects each disk of $\operatorname{cl}(\operatorname{int}(H) \cap F)$ at exactly one
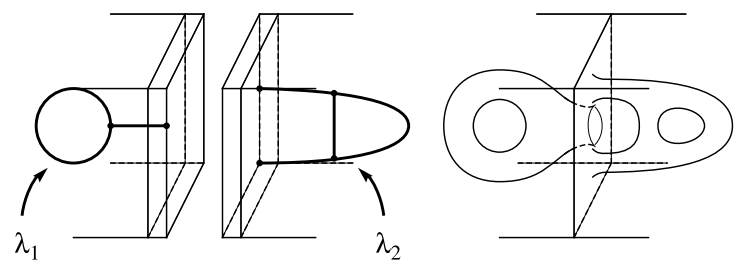

Figure 3: Amalgamation of Heegaard splittings. 
point and has no other intersections with the boundary of this handlebody. Since the two handlebodies were obtained from $H$ by compressing along the disks $\operatorname{cl}(\operatorname{int}(H) \cap F)$, it is easy to construct a spine for $H$ by connecting $\lambda_{2}$ to $\mu_{1} . H^{\prime}$ is treated similarly; the proposition follows.

\section{Proof of Theorem 1.1}

We adopt the notations of Section 2 .

Let $3_{1}$ be the trefoil knot and $4_{1}$ the figure eight knot. Let $L=l_{1} \cup l_{2}$ be a hyperbolic two-bridge link. Denote $\partial N\left(l_{i}\right)$ by $T_{i}(i=1,2)$.

We note that there exists an essential annulus $\bar{A}$ in $E\left(3_{1}\right)$ such that the closures of the components of $E\left(3_{1}\right) \backslash \bar{A}$ are solid tori, say $N_{1}$ and $N_{1}^{\prime}$, where $\bar{A}$ wraps around $N_{1}$ longitudinally twice and around $N_{1}^{\prime}$ longitudinally three times. Hence, $N_{1} \cap \partial E\left(3_{1}\right)$ and $N_{1}^{\prime} \cap \partial E\left(3_{1}\right)$ are incompressible and boundary incompressible. On the other hand, we note that $4_{1}$ is a genus 1 fibered knot. Hence we have the following: let $S \subset E\left(4_{1}\right)$ be a minimal genus Seifert surface for $4_{1}$ (note that $S$ is a once punctured torus). Let $N_{2}=N(S)$ and $N_{2}^{\prime}=\operatorname{cl}\left(E\left(4_{1}\right) \backslash N_{2}\right)$. Then $N_{2}$ ( $N_{2}^{\prime}$ resp.) is homeomorphic to $S \times[0,1]$, where $N_{2} \cap \partial E\left(4_{1}\right)\left(N_{2}^{\prime} \cap \partial E\left(4_{1}\right)\right.$ resp.) corresponds to $\partial S \times[0,1]$. Note that $S \times[0,1]$ is homeomorphic to a genus 2 handlebody, and $\partial S \times[0,1]$ is incompressible and $\partial$-incompressible in $S \times[0,1]$. Let $P$ be a bridge sphere in $E(L)$. Then as in Section 2, $P$ separates $E(L)$ into two genus 2 handlebodies, called $H$ and $H^{\prime}$. Finally, let $M$ be a three-manifold obtained from $E\left(3_{1}\right) \cup E\left(4_{1}\right)$ and $E(L)$ by identifying their boundaries by a homeomorphism $h:\left(\partial E\left(3_{1}\right) \cup \partial E\left(4_{1}\right)\right) \rightarrow \partial E(L)\left(=T_{1} \cup T_{2}\right)$, so that $h$ satisfies the following conditions:

(1) $h\left(N_{1} \cap \partial E\left(3_{1}\right)\right)=H \cap T_{1}$, hence $h\left(N_{1}^{\prime} \cap \partial E\left(3_{1}\right)\right)=H^{\prime} \cap T_{1}$.

(2) $h\left(N_{2} \cap \partial E\left(4_{1}\right)\right)=H \cap T_{2}$, hence $h\left(N_{2}^{\prime} \cap \partial E\left(4_{1}\right)\right)=H^{\prime} \cap T_{2}$.

Note that the conditions of Proposition 2.1 are satisfied, and so we see that $M$ admits a strongly irreducible genus 3 Heegaard splitting. Explicitly, the splitting surface is obtained from the bridge sphere $P$ by attaching $\operatorname{Fr}_{E\left(4_{1}\right)} N_{2}$ (that is, two once-punctured tori) in $E\left(4_{1}\right)$ and $\bar{A}$ in $E\left(3_{1}\right)$. Denote this splitting by $V \cup_{\Sigma} V^{\prime}$, where $V$ and $V^{\prime}$ are the handlebodies of $N_{1} \cup H \cup N_{2}$ and $N_{1}^{\prime} \cup H^{\prime} \cup N_{2}^{\prime}$, respectively, and $\Sigma$ is the splitting surface.

The decomposition $E\left(3_{1}\right) \cup E(L) \cup E\left(4_{1}\right)$ is the torus decomposition for $M$. In [4, Theorem], a complete list of Heegaard genus 2 three-manifolds admitting non-trivial torus decomposition is given. By consulting that list, we see that $g(M)>2$. Above we constructed a strongly irreducible genus 3 
Heegaard splitting for $M$. We conclude that $g(M)=3$, and that $M$ admits a strongly irreducible minimal genus Heegaard splitting.

We claim that the sub-manifold $E\left(3_{1}\right) \cup E(L)$ admits a genus 2 Heegaard splitting. Since $A_{1}$ is primitive in $H$ and $A_{1}^{\prime}$ is primitive in $H^{\prime}, N_{1} \cup H$ and $N_{1}^{\prime} \cup H^{\prime}$ are genus 2 handlebodies. Let $A=H \cap T_{2}$ and $A^{\prime}=H^{\prime} \cap T_{2}$. Let $C=\operatorname{cl}\left(\left(N_{1} \cup H\right) \backslash N(A, H)\right)$ and $C^{\prime}=\left(N_{1}^{\prime} \cup H^{\prime}\right) \cup N(A, H)$. It is clear that $C$ is a genus 2 handlebody. It is easy to see that $A^{\prime}$ is primitive in $N_{1}^{\prime} \cup H^{\prime}$, i.e., there is a meridian disk $\Delta^{\prime}$ of $N_{1}^{\prime} \cup H^{\prime}$ such that $\Delta^{\prime} \cap A^{\prime}$ is an essential arc in $A^{\prime}$. This implies that $C^{\prime}$ is a genus 2 compression body with $\partial_{-} C^{\prime}=A \cup A^{\prime}=T_{2}$. Denoting $\partial_{+} C$ by $\Sigma^{\prime}$, we see that $C \cup_{\Sigma^{\prime}} C^{\prime}$ is a genus 2 Heegaard splitting of $E\left(3_{1}\right) \cup E(L)$.

Remark 3.1. For future reference, we note the following: Let $\alpha$ be a core curve of the solid torus $N_{1}$ and $\alpha^{\prime}$ a core curve of the solid torus $N_{1}^{\prime}$. By construction, $\alpha$ is contained in a spine of the handlebody $C$ and $\alpha^{\prime}$ is contained in a spine of the compression body $C^{\prime}$. Similarly, the decomposition $M=\bar{C} \cup \bar{C}^{\prime}$, where $\bar{C}=\left(N_{1} \cup H\right) \cup N\left(A^{\prime}, H^{\prime}\right)$ and $\bar{C}^{\prime}=\operatorname{cl}\left(\left(N_{1}^{\prime} \cup H^{\prime}\right) \backslash\right.$ $N\left(A^{\prime}, H^{\prime}\right)$ ), gives another (possibly isotopic) genus 2 Heegaard splitting of $E\left(3_{1}\right) \cup E(L)$ so that $\alpha^{\prime}$ is contained in a spine of the handlebody $\bar{C}^{\prime}$ and $\alpha$ is contained in a spine of the compression body $\bar{C}$.

It is well known that $E\left(4_{1}\right)$ admits a genus 2 Heegaard splitting. By amalgamating a genus 2 Heegaard splitting for $E\left(4_{1}\right)$ with a genus 2 Heegaard splitting of $E\left(3_{1}\right) \cup E(L)$, we obtain a weakly reducible Heegaard splitting of $M$; by Schultens [10, Remark 2.7] (see also [7, Lemma 2.7] for a more general statement) this Heegaard splitting has genus 3 . This establishes the existence of weakly reducible minimal genus Heegaard splittings of $M$.

This completes the proof of Theorem 1.1.

Remark 3.2. The following is a suggestion for a way to generalize the results of this paper. Fix $g \geq 3$. Let $H$ (resp. $H^{\prime}$ ) be a genus $g-1$ handlebody and $A_{1}, A_{2} \subset \partial H$ (resp. $A_{1}^{\prime}, A_{2}^{\prime} \subset \partial H^{\prime}$ ) be two primitive annuli. Similar to the construction above, identify $\operatorname{cl}\left(\partial H \backslash\left(A_{1} \cup A_{2}\right)\right)$ with $\operatorname{cl}\left(\partial H^{\prime} \backslash\left(A_{1}^{\prime} \cup\right.\right.$ $\left.\left.A_{2}^{\prime}\right)\right)$. To the resulting manifold, glue $E\left(3_{1}\right)$ and $E\left(4_{1}\right)$ in a way that $\partial H \backslash$ $\left(A_{1} \cup A_{2}\right)$ union two fibers of $E\left(4_{1}\right)$ union an essential annulus of $E\left(3_{1}\right)$ gives a genus $g$ Heegaard splitting, say $V \cup V^{\prime}$.

The curve complex distance of a Heegaard splitting was defined by Hempel [3] and was generalized by several authors to bridge decompositions; note that $H \cup H^{\prime}$ is a genus $g-3$, two-bridge decomposition (the link in question is the core of the attached solid tori when filling $H \cup H^{\prime}$ along 
the slope defined by $H \cap H^{\prime}$; see, for example, the proof of Proposition 2.2 of [8], where we defined generalized bridge decomposition in terms of a surface with boundary in the link exterior). It is reasonable to expect that if the distance of $H \cup H^{\prime}$ is large, then $V \cup V^{\prime}$ is strongly irreducible and minimal genus (Tomova's [11] should be useful here). Similar to the construction above, one obtains weakly reducible minimal genus Heegaard splittings by considering the decomposition $E\left(3_{1}\right) \cup H \cup H^{\prime}$ and $E\left(4_{1}\right)$. This would give manifolds of genus $g$, for arbitrary $g \geq 3$, admitting both weakly reducible and strongly irreducible minimal genus Heegaard splittings.

\section{Further examples: the bounded case}

Throughout this section, let $M=E\left(3_{1}\right) \cup E(L) \cup E\left(4_{1}\right)$ be any of the manifolds constructed in the previous section. Let $V \cup_{\Sigma} V^{\prime}$ be the strongly irreducible Heegaard splitting constructed there.

Let $\beta^{*} \subset E\left(4_{1}\right)$ be the simple, closed curve given in Figure 4. By Figure $4(\mathrm{a}), \beta^{*}$ is contained in a once-punctured torus that is a fiber of the fibration of $E\left(4_{1}\right)$ over $S^{1}$. We may choose this fiber to be a component of $\Sigma \cap E\left(4_{1}\right)$.

Remark 4.1. We connect $\beta^{*}$ to $\partial E\left(4_{1}\right)$ by an arc as in Figure 4(b). By using slideisotopy, we see that the exterior of a regular neighborhood of $\left(\partial E\left(4_{1}\right)\right.$ together with the one-complex) is a genus 2 handlebody (see, for example, [5, Figures 5 and 6$]$ ). This shows that $\beta^{*}$ is contained in a spine of a compression body (not handlebody) component of a genus 2 Heegaard splitting of $E\left(4_{1}\right)$.

Let $\alpha, \alpha^{\prime}$ be as in Remark 3.1, so that $\alpha \subset V$ and $\alpha^{\prime} \subset V^{\prime}$. Denote $\operatorname{cl}\left(M \backslash N\left(\alpha \cup \beta^{*} \cup \alpha^{\prime}\right)\right)$ by $X$. Denote the boundary components of $X$ by $T_{\alpha}=\partial N(\alpha), T_{\beta^{*}}=\partial N\left(\beta^{*}\right)$ and $T_{\alpha^{\prime}}=\partial N\left(\alpha^{\prime}\right)$.

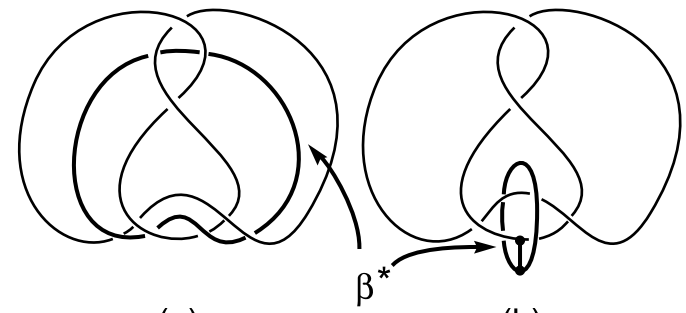

(a)

(b)

Figure 4: $4_{1}, \beta^{*}$, and the arc connecting them. 
Lemma 4.1. $X$ admits two genus three weakly reducible Heegaard surfaces, denoted by $F_{1}$ and $F_{2}$, so that

(1) $F_{1}$ separates $T_{\alpha} \cup T_{\beta^{*}}$ and $T_{\alpha^{\prime}}$.

(2) $F_{2}$ separates $T_{\alpha}$ and $T_{\alpha^{\prime}} \cup T_{\beta^{*}}$.

Proof. By applying Proposition 2.2 to the Heegaard splitting $C \cup C^{\prime}$ (recall Remark 3.1) and the genus 2 Heegaard splitting of $E\left(4_{1}\right)$ given in Remark 4.1 we obtain a genus 3 Heegaard splitting of $M$ such that the Heegaard surface separates $\alpha \cup \beta^{*}$ and $\alpha^{\prime}, \alpha \cup \beta^{*}$ is contained in a spine of one of the handlebodies and $\alpha^{\prime}$ is contained in a spine of the other handlebody. This gives $F_{1}$.

Analogously, by applying Proposition 2.2 to the Heegaard splitting $\bar{C} \cup$ $\bar{C}^{\prime}$ (recall Remark 3.1) and the genus 2 Heegaard splitting of $E\left(4_{1}\right)$ given in Remark 4.1 we obtain $F_{2}$.

Lemma 4.2. $g(X)=3$.

Proof. Since $M$ is obtained from $X$ by Dehn filling, we have that $g(X) \geq$ $g(M)=3$. On the other hand, $F_{1}$ is a genus 3 Heegaard surface for $X$, showing that $g(X) \leq g\left(F_{1}\right)=3$.

Definition 4.1. Let $C$ be a compression body and $\alpha_{1}, \ldots, \alpha_{n} \subset C$ simple closed curves. We say that $\alpha_{1}, \ldots, \alpha_{n}$ are simultaneous cores, if the following two conditions hold:

(1) There exist mutually disjoint annuli $A_{1}, \ldots, A_{n} \subset C$ so that one component of $\partial A_{i}$ is $\alpha_{i}$ and the other is on $\partial_{+} C$.

(2) There exist mutually disjoint meridian disks $D_{1}, \ldots, D_{n} \subset C$ so that $\alpha_{i}$ intersects $D_{i}$ transversely at one point and for $i \neq j, \alpha_{i} \cap D_{j}=\emptyset$.

Remark 4.2. It is easy to see that $\alpha_{1}, \ldots, \alpha_{n} \subset C$ are simultaneous cores if and only if $\operatorname{cl}\left(C \backslash N\left(\cup_{i=1}^{n} \alpha_{i}\right)\right)$ is a compression body.

Recall that $\beta^{*} \subset \Sigma \cap E\left(4_{1}\right)$. Let $\beta$ (resp. $\beta^{\prime}$ ) be a curve obtained by pushing $\beta^{*}$ slightly into $V$ (resp. $V^{\prime}$ ).

Lemma 4.3. The curves $\alpha, \beta \subset V$ and $\alpha^{\prime}, \beta^{\prime} \subset V^{\prime}$ are simultaneous cores.

Proof. Recall the definition of the handlebody $H=V \cap E(L)$ given in Section 2 , and let $\Delta_{1}$ and $\Delta_{2}$ be the meridian disks of $H$ shown in Figure 1. Let 
$\widehat{D}_{\alpha}$ be a meridian disk of the solid torus $N_{1}=V \cap E\left(3_{1}\right)$ that intersects the annulus $\bar{A}=\Sigma \cap E\left(3_{1}\right)$ essentially. By attaching two copies of $\Delta_{1}$ to $\widehat{D}_{\alpha}$, we obtain a meridian disk for $V$, denoted by $D_{\alpha}$, that intersects $\alpha$ once and is disjoint from $\beta$.

Recall that $V \cap E\left(4_{1}\right)\left(=N_{2}\right)$ is homeomorphic to $S \times[0,1]$, where $S$ is a once-punctured torus. We may suppose that $\beta$ corresponds to a curve $\beta_{S} \times\{1 / 2\}$, where $\beta_{S}$ is an essential curve on $S$. Let $\widehat{D}_{\beta}$ be a vertical disk in $V \cap E\left(4_{1}\right)$ that intersects $\beta$ once, that is, $\widehat{D}_{\beta}$ corresponds to a disk of the form $\gamma \times[0,1]$, where $\gamma$ is an arc properly embedded in $S$ that intersects $\beta_{S}$ transversely once. By attaching two copies of $\Delta_{2}$ to $\widehat{D}_{\beta}$, we obtain a meridian disk for $V$, denoted by $D_{\beta}$, that intersects $\beta$ once and is disjoint from $\alpha$. It is easy to see that $D_{\alpha} \cap D_{\beta}=\emptyset$.

The annulus $A_{\beta}=\beta_{S} \times[1 / 2,1]$ is embedded in $V$, with one boundary component $\beta$ and the other on $\partial V$. Let $\widehat{A}_{\alpha}$ be an annulus embedded in $N_{1}$ with one boundary component $\alpha$ and the other on $\partial N_{1}$, which intersects the annulus $\bar{A}$ at three essential arcs. By attaching three copies of $\Delta_{1}$ to $\widehat{A}_{\alpha}$, we obtain an annulus $A_{\alpha}$ embedded in $V$, with one boundary component $\alpha$ and the other on $\partial V$. By construction, $A_{\alpha} \cap A_{\beta}=\emptyset$.

Using $D_{\alpha}, D_{\beta}, A_{\alpha}$ and $A_{\beta}$, we see that $\alpha$ and $\beta$ are simultaneous cores. The curves $\alpha^{\prime}$ and $\beta^{\prime}$ are treated similarly.

Theorem 4.1. For $i=1,2,3$, there exists infinitely many manifolds $M_{i}$ so that $\partial M_{i}$ consists of exactly $i$ tori, $g\left(M_{i}\right)=3$, and each $M_{i}$ admits both strongly irreducible and weakly reducible minimal genus Heegaard splittings.

Moreover, each manifold $M_{2}$ admits four distinct minimal genus Heegaard surfaces, denoted as $F_{\mathrm{SI}}^{1,1}, F_{\mathrm{WR}}^{1,1}, F_{\mathrm{SI}}^{2,0}, F_{\mathrm{WR}}^{2,0}$, so that the following four conditions hold:

(1) The Heegaard splittings given by $F_{\mathrm{SI}}^{1,1}$ and $F_{\mathrm{SI}}^{2,0}$ are strongly irreducible.

(2) The Heegaard splittings given by $F_{\mathrm{WR}}^{1,1}$ and $F_{\mathrm{WR}}^{2,0}$ are weakly reducible.

(3) $F_{\mathrm{SI}}^{1,1}$ and $F_{\mathrm{WR}}^{1,1}$ separate the two boundary components of $M_{2}$.

(4) $F_{\mathrm{SI}}^{2,0}$ and $F_{\mathrm{WR}}^{2,0}$ do not separate the boundary components of $M_{2}$.

Before proving Theorem 4.1, we give the following definition.

Definition 4.2. Let $Y_{1}$ and $Y_{2}$ be manifolds so that $Y_{1}$ is obtained from $Y_{2}$ by Dehn filling (equivalently, $Y_{2}$ is obtained from $Y_{1}$ by removing an open regular neighborhood of a link in it). Note that $Y_{2} \subset Y_{1}$. 
Let $\Sigma_{2} \subset Y_{2}$ be any Heegaard surface. Then $\Sigma_{2}$ is a Heegaard surface of $Y_{1}$. We say that $\Sigma_{2} \subset Y_{1}$ is an induced Heegaard surface (or the Heegaard surface induced by $\Sigma_{2}$ ).

Let $\Sigma_{1} \subset Y_{1}$ be a Heegaard surface. Suppose that $\Sigma_{1} \subset Y_{2}$ and that $\Sigma_{1}$ is a Heegaard surface of $Y_{2}$. We say that $\Sigma_{1} \subset Y_{2}$ is an induced Heegaard surface (or the Heegaard surface induced by $\Sigma_{1}$.)

The proof of the following lemma is easy and left to the readers:

Lemma 4.4. Let $Y_{1}$ and $Y_{2}$ be as above. If a Heegaard surface $\Sigma_{2}$ of $Y_{2}$ is weakly reducible, then so is the induced Heegaard surface. On the other hand, if $\Sigma_{1} \subset Y_{1}$ is a strongly irreducible Heegaard surface that induces a Heegaard surface for $Y_{2}$, then the induced Heegaard surface is strongly irreducible.

Proof of Theorem 4.1. We deal with the cases $i=1,2$ and 3 in increasing order of difficulty.

For $i=3$, let $M_{3}=X$. Then by Lemmas 4.1 and $4.2, g(X)=3$ and $X$ admits a weakly reducible minimal genus Heegaard splitting.

Note that $\beta^{*}$ is isotopic to $\beta$; hence $X$ is homeomorphic to $\operatorname{cl}(M \backslash$ $\left.N\left(\alpha \cup \alpha^{\prime} \cup \beta\right)\right)$. By Lemma 4.3 and Remark 4.2, $V \cup V^{\prime}$ induces a genus 3 Heegaard splitting of $\operatorname{cl}\left(M \backslash N\left(\alpha \cup \alpha^{\prime} \cup \beta\right)\right)$. Since $V \cup V^{\prime}$ is strongly irreducible, Lemma 4.4 shows that the induced Heegaard splitting is strongly irreducible. The case $i=3$ follows.

For $i=1$, let $M_{1}=\operatorname{cl}(M \backslash N(\alpha))$. Then $g\left(M_{1}\right) \geq g(M)=3$. Since $X$ is obtained from $M_{1}$ by removing an open neighborhood of $\alpha^{\prime}$ and $\beta^{*}, g\left(M_{1}\right) \leq$ $g(X)=3$. We see that $g\left(M_{1}\right)=3$.

Note that $M_{1}$ is obtained by filling two boundary components of $X$. Hence the genus 3 weakly reducible Heegaard splittings for $X$ given in Lemma 4.1 induces genus 3 weakly reducible Heegaard splittings for $M_{1}$.

By Lemma 4.3 and Remark 4.2, $V \cup V^{\prime}$ induces a genus 3 Heegaard splitting for $M_{1}$. As above, the induced Heegaard splitting is strongly irreducible. The case $i=1$ follows.

For $i=2$, let $M_{2}=\operatorname{cl}\left(M \backslash N\left(\alpha \cup \beta^{*}\right)\right)$. Similar to $M_{1}$, it is easy to see that $g\left(M_{2}\right)=3$.

By Lemma 4.4, each of the two genus 3 weakly reducible Heegaard splittings given in Lemma 4.1 induces a genus 3 weakly reducible Heegaard splitting on $M_{2}$, one not separating the components of $\partial M_{2}$ (corresponding to Lemma 4.1(1)), and the other separating them (corresponding to Lemma $4.1(2))$. These are the surfaces $F_{\mathrm{WR}}^{2,0}$ and $F_{\mathrm{WR}}^{1,1}$ in the theorem. 
Note that $\beta$ ( $\beta^{\prime}$ resp.) is isotopic to $\beta^{*}$; hence, $M_{2}$ is homeomorphic to $\operatorname{cl}(M \backslash N(\alpha \cup \beta))\left(\operatorname{cl}\left(M \backslash N\left(\alpha \cup \beta^{\prime}\right)\right)\right.$ resp. $)$. By Lemma 4.3 and Remark 4.2, $V \cup V^{\prime}$ induces a Heegaard splitting for $\operatorname{cl}(M \backslash N(\alpha \cup \beta))$ that does not separate the boundary components of $\operatorname{cl}(M \backslash N(\alpha \cup \beta))$. The corresponding Heegaard surface for $M_{2}$ is the surface $F_{\mathrm{SI}}^{2,0}$. Similarly, by Lemma 4.3 and Remark 4.2, $V \cup V^{\prime}$ induces a Heegaard splitting for $\operatorname{cl}\left(M \backslash N\left(\alpha \cup \beta^{\prime}\right)\right)$ that separates the boundary components of $\operatorname{cl}\left(M \backslash N\left(\alpha \cup \beta^{\prime}\right)\right)$. The corresponding Heegaard surface for $M_{2}$ is the surface $F_{\mathrm{SI}}^{1,1}$. By Lemma $4.4, F_{\mathrm{SI}}^{2,0}$ and $F_{\text {SI }}^{1,1}$ are strongly irreducible. The case $i=2$ follows.

\section{Acknowledgment}

T. Kobayashi was supported by Grant-in-Aid for scientific research, JSPS grant number 19540083. Y. Rieck was supported in part JSPS (fellow number P00024) and by the 21st century COE program "Constitution for wideangle mathematical basis focused on knots" (Osaka City University); leader: Akio Kawauchi.

\section{References}

[1] A.J. Casson and C.McA. Gordon, Reducing Heegaard splittings, Topol. Appl. 27(3) (1987), 275-283. MR 89c:57020.

[2] W. Haken, Some results on surfaces in 3-manifolds, Studies in modern topology, Math. Assoc. Am. Prentice-Hall, Englewood Cliffs, NJ, 1968, 39-98, MR MR0224071 (36 \#7118).

[3] J. Hempel, 3-manifolds as viewed from the curve complex, Topology 40 (2001), 631-657.

[4] T. Kobayashi, Structures of the Haken manifolds with Heegaard splittings of genus two, Osaka J. Math. 21(2) (1984), 437-455. MR 85k:57011.

[5] T. Kobayashi, A criterion for detecting inequivalent tunnels for a knot, J. Math. Proc. Cambridge Philos. Soc. 107(3) (1990), 483-491.

[6] T. Kobayashi, A construction of 3-manifolds whose homeomorphism classes of Heegaard splittings have polynomial growth, Osaka J. Math. 29(4) (1992), 653-674. MR 93j:57007. 
[7] T. Kobayashi and Y. Rieck, Heegaard genus of the connected sum of msmall knots, Commun. Anal. Geom. 14(5) (2006), 1033-1073, Preprint available at arxiv.org/abs/math.GT/0503229.

[8] T. Kobayashi and Y. Rieck, Knot exteriors with additive Heegaard genus and Morimoto's conjecture, Algebr. Geom. Topol. 8(2) (2008), 953-969, Preprint available at arxiv.org/abs/math.GT/0701765v2.

[9] Y. Moriah, Connected sums of knots and weakly reducible Heegaard splittings, Topol. Appl. 141(1-3) (2004), 1-20. MR 2058679.

[10] J. Schultens, The classification of Heegaard splittings for (compact orientable surface) $\times S^{1}$, Proc. London Math. Soc. (3) 67(2) (1993), 425448. MR MR1226608 (94d:57043).

[11] M. Tomova, Multiple bridge surfaces restrict knot distance, Algebr. Geom. Topol. 7 (2007), 957-1006, Preprint available at arxiv.org/abs/math.GT/0511139.

Department of Mathematics

NARA WOMEN's UNIVERSITY

Kitauoya Nishimachi

NARA 630-8506

JAPAN

E-mail address: tsuyoshi@cc.nara-wu.ac.jp

Department of Mathematical Sciences

UNIVERSITY OF ARKANSAS

FAYETTEVILLE

AR 72701

USA

E-mail address: yoav@uark.edu

Received December 30, 2008 
